\title{
JOINT IMAGE HALFTONING AND WATERMARKING IN HIGH-RESOLUTION DIGITAL FORM
}

\author{
Chao-Yong Hsu and Chun-Shien Lu
}

\author{
Institute of Information Science, Academia Sinica, Taipei, Taiwan, ROC
}

\begin{abstract}
The existing halftone image watermarking methods were proposed to embed a watermark bit in a halftone dot, which corresponds to a pixel, to generate stego halftone image. This one-to-one mapping, however, is not consistent with the one-to-many strategy that is used by current high-resolution devices, such as computer printers and screens, where one pixel is first expanded into many dots and then a halftoning processing is employed to generate a halftone image. Furthermore, electronic paper or smart paper that produces high-resolution digital files cannot be protected by the traditional halftone watermarking methods. In view of these facts, we present a high-resolution halftone watermarking scheme to deal with the aforementioned problems. The characteristics of our scheme include: (i) a high-resolution halftoning process that employs a one-to-many mapping strategy is proposed; (ii) a many-to-one inverse halftoning process is proposed to generate gray-scale images of good quality; and (iii) halftone image watermarking can be directly conducted on gray-scale instead of halftone images to achieve better robustness.
\end{abstract}

\section{INTRODUCTION}

Halftoning [6] refers to the physical process of converting a continuous tone images to black and white dots, as shown in Fig. 1. With the advent of digitization and population of bi-level devices such as printers and fax machines, digital halftoning techniques become indispensable. In addition, since digital right management has received much attention, protection of halftone images is an important topic that cannot be ignored. This paper will focus on digital halftoning image watermarking.

Over the past few years, some halftone image watermarking methods have been proposed $[2,3,11]$. We found that their common characteristic is that the target for protection is a low-resolution printed paper. Under this circumstance, digital halftoning techniques [6] such as order dithering or error diffusion are employed to generate halftone images. However, we have an open question: "Is printed

Corresponding author: Dr. C. S. Lu (lcs@iis.sinica.edu.tw)

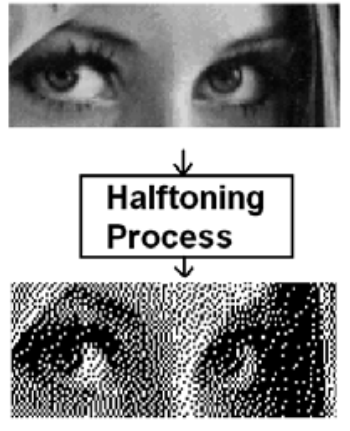

Fig. 1. Digital halftoning by transforming gray-scale into bilevel.

form beneficial to be distributed and, thus, worthy protected by means of digital watermarking?" In addition, we pose another question: "What is the resolution of such a halftone image?" These questions were, unfortunately, ignored in the past and we shall answer them in the study. Let us take a computer screen of 19 inches and a displayed image of size $1280 \times 1024$ as examples for explanation. When a pixel is converted as a dot ${ }^{1}$, this implies that the resolution is $86\left(=\frac{1280}{14.82}\right)$ dpi (dots per inch), where 14.82 inch denotes the row's length corresponding to 1280 dots. We note that $86 \mathrm{dpi}$ is so low that poor quality will be perceived if the low-resolution halftone image is displayed on the screen. We also note that even the current printers or scanners are equipped with the lowest resolution higher than 100 dpi. As a result, it is impractical to perform digital watermarking on halftone images of low resolutions because no device can be found to support them.

Based on the above reasons, a novel high-resolution digital halftoning image watermarking scheme is presented in this paper. Our work is also motivated by the recent development of a new display device, called "electronic paper (e-paper)" [1] or "smart paper," which can get a digital file from a personal computer through I/O port and display it without needing power. During the past few years,

\footnotetext{
${ }^{1}$ This one-to-one mapping is adopted in current halftone watermarking methods.
} 
many companies such as Philips, eInk, Sony, and Xerox have devoted to manufacturing the first high-resolution electronic ink-based display module for reading-intensive applications. In addition, electronic paper is advantageous to environments in that no any tree is hewn down during the generation process. Thus, our concern is that the existing low-resolution halftone image watermarking methods do not meet the requirement of e-paper.

In the literature, the existing halftone image watermarking technologies can be divided into two categories according to where the watermarking process is done. The methods in the first category are presented to embed watermarks during the halftoning process [3], while the second category includes those that directly embed watermarks into a halftone image [11, 12]. However, watermark is difficult to be detected from attacked halftone image since one-toone halftoning process is used, so that even slight geometric distortions can destroy this kind of mapping. Since the socalled "high-resolution halftone image" is considered in this study, the proposed many-to-one inverse halftoning is effective to produce a good quality of inverse halftoned (grayscale) images. Thus, this feature is beneficial for us to perform watermark embedding and detection in gray-scale images by directly exploiting robust gray-scale image watermarking methods.

Therefore, the major contributions of this study are twofold: (i) a high-resolution digital halftoning technique is presented, so that the digital files used in the device, e-paper, can be protected by our method; and (ii) based on (i), a robust grayscale image watermarking method (e.g., $[7,8])$ can be directly exploited to achieve robust halftoning watermarking.

\section{PROPOSED METHOD}

In this study, a watermarked halftone image is generated by embedding watermarks into a gray-scale images, which is then transferred as a stego halftone images by means of a proposed high-resolution halftoning process. Block diagram of our method is depicted in Fig. 2.

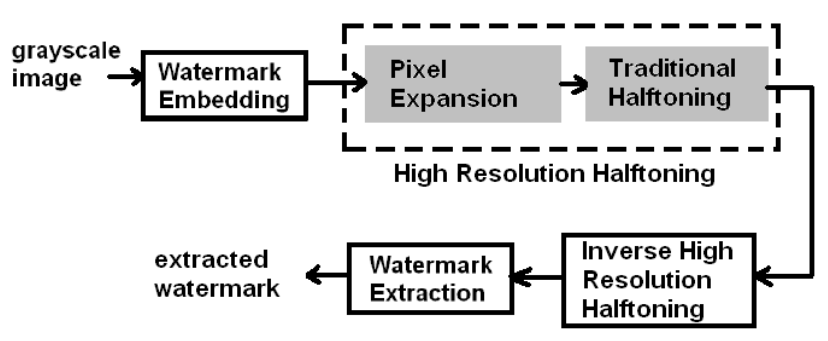

Fig. 2. Block diagram of the proposed high-resolution digital halftoning watermarking scheme.

\subsection{Gray-Scale Image Watermarking}

Our previous block-based content-dependent watermarking method [7] is adopted due to its robustness against contentpreserving modifications. For each gray-scale image, it is divided into macroblocks of size $M \times 2 M$ (containing two $M \times M$ blocks), as shown in Fig. 3. In a macroblock, a watermark sequence of 64 bits is embedded for both content protection and authentication. In this way, a periodical signal composed of several watermarks is concealed. In our scheme, a macroblock/block is the minimum unit, where content protection/authentication can be determined.

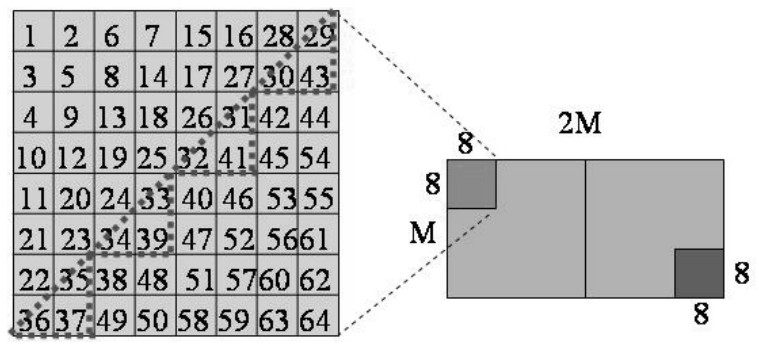

Fig. 3. Block relationship $(M=32)$ : one watermark bit is embedded in a pair of triangular areas in a block pair.

At the embedding stage, each $M \times M$ block is further divided into $8 \times 8$ blocks (denoted as $B_{8}(\cdot)$ ) and each $8 \times 8$ block pair is embedded with 4 watermark bits. With our approach, a watermark bit is embedded by forcing a relationship between the $\mathrm{AC}$ energies of a block pair, $B_{8}(i)$ and $B_{8}(j)$, as follows: (i) if " 1 " is embedded, let $A C_{\text {eng }}\left(B_{8}(i)\right)>$ $A C_{\text {eng }}\left(B_{8}(j)\right)$; (ii) if " 0 " is embedded, let $A C_{\text {eng }}\left(B_{8}(i)\right)<$ $A C_{\text {eng }}\left(B_{8}(j)\right)$. The $\mathrm{AC}$ energy in a $8 \times 8$ block is defined to be the magnitude summation of the $\mathrm{AC}$ coefficients selected within a dashed triangular area, as shown in Fig. 3. In addition, the block pairs in an $M \times M$ block is determined by a secret key, which is the same in generating a watermark. At the detection stage, the hidden bit is extracted by checking the above relationship between a pair of blocks.

For copyright protection, all the watermarks extracted from $M \times 2 M$ blocks can be integrated to get the final but more accurate estimation. This operation is similar to collusion but is performed from an owner's viewpoint. As we have studied in $[7,8]$, this is a very efficient step in accurately estimating the hidden watermark.

\subsection{Generation of High-Resolution Halftone Images}

After the stego image has been yielded, it needs to be used to generate a high-resolution halftone image to meet the goal of halftone image distribution. The resolution of a halftone image can be defined as the number of dots per inch (dpi). A dot may be represented by a block of $B \times B$ pixels for AM halftoning or represented by only one pixel for FM 
halftoning. To achieve this, the expected size of a digital high-resolution halftone image must be set in advance before halftoning. In this study, we adopt AM halftoning because it has been used by current printers. Let $X \times Y$ be the size of a stego image and let a halftone image of size $P$ inch $\times Q$ inch with $s$ dpi be displayed over a screen, which implies a size of $(B \times P \times s)$ pixel $\times(B \times Q \times s)$ pixel. Under this circumstance, a pixel of a stego image is expanded to an area of $\frac{(B \times P \times s)}{X} \times \frac{(B \times Q \times s)}{Y}$ pixels for AM halftoning ${ }^{2}$; i.e., the expanded stego image is of size $(B \times P \times s) \times(B \times Q \times s)$ ${ }^{3}$. After the aforementioned mapping has been performed, the resized stego image is then halftoned by means of cluster dot dithering [4] to yield a stego halftone image.

\subsection{Inverse Halftoning of A High-Resolution Halftone Image for Watermark Detection}

The existing techniques of inverse halftoning such as lookup table $[9,10]$ and filtering $[5,13]$ cannot yield good quality of inverse halftone images without raising perceptual degradation. The main reason is that a halftone image has lost a great deal of information from its original gray-scale image during the halftoning processing so that a halftone pixel (either 0 or 1 ) cannot find its original pixel. Particularly, this property is harmful to watermark detection that is performed on gray-scale images. However, since the proposed high-resolution halftoning has expanded the size of an image, more available information can be used to reconstruct the inverse halftone image; i.e., a block of $B \times B$ pixels corresponds to an original pixel. Specifically, for each block of size $B \times B$ in a halftone image, the number $W$ of white pixels is calculated, where $0 \leq W \leq B^{2}$. Then, a gray-scale is calculated as $\left\lfloor\frac{W}{B^{2}} \times 255\right\rfloor$. By repeating the above process, a gray-scale inverse halftone image of good quality can be generated.

\section{EXPERIMENTAL RESULTS}

In this section, two sets of experiments were, respectively, conducted to demonstrate the excellent performance of the proposed high-resolution inverse halftoning process, and grayscale image watermarking and halftoning for watermark detection. In our experiments, ten varieties of gray-scale images of size $512 \times 512$ were used. The block size used for AM halftoning, as described in Sec. 2.2, was $11 \times 11$. Three different resolutions, i.e., $100 \mathrm{dpi}, 200 \mathrm{dpi}$, and 300 dpi were adopted to evaluate our method.

\footnotetext{
${ }^{2}$ In current halftone watermarking methods, $B \times P \times s=X$ and $B \times Q \times s=Y$.

${ }^{3}$ This implies that a digital file stored in an e-paper device needs more storages.
}

Table 1. Quality comparisons between original image, and its halftoned and then inversely halftoned version

\begin{tabular}{|c||ccc|}
\hline \multicolumn{1}{|c||}{} & \multicolumn{3}{c|}{ Resolution } \\
Images & 100dpi & 200dpi & 300dpi \\
\hline Splash & 34.85 & 40.47 & 43.41 \\
\hline Tank & 34.23 & 39.52 & 42.36 \\
\hline Lena & 33.24 & 38.51 & 41.34 \\
\hline Baboon & 24.79 & 30.09 & 33.21 \\
\hline Bridge & 28.47 & 33.81 & 36.89 \\
\hline Clock & 34.01 & 39.66 & 42.80 \\
\hline F16 & 32.97 & 38.64 & 41.61 \\
\hline Pepper & 30.11 & 35.16 & 38.12 \\
\hline Sailboat & 29.49 & 34.70 & 37.65 \\
\hline Goldhill & 32.68 & 38.18 & 41.08 \\
\hline
\end{tabular}

\subsection{Image Quality of High-Resolution Inverse Halfton- ing}

Image quality of the proposed inverse high-resolution halftoning technique was evaluated in terms of PSNR measured between an original image, and its corresponding halftoned and then inversely halftoned version. The obtained results are depicted in Table 1. We observe from Table 1 that the complex image, Baboon, easily leads to lower PSNR values. For perceptual quality comparison, a pair of smoothing images, the original pepper and the inversely high-resolution halftoned pepper obtained at $200 \mathrm{dpi}$, are shown in Fig. 4 , where no perceptual difference can be perceived. We also note that traditional low-resolution inverse halftoning $[5,9,10]$ can only obtain PSNRs $\leq 30 \mathrm{~dB}$.

\subsection{Gray-scale Image Watermarking and Halftoning for Watermark Detection}

The proposed watermarking method, as shown in Fig. 2, was used to generate stego halftone images. In this experiment, each image block was of size $32 \times 32$ so that the watermark's length was 32 bits and the number of blocks was 256. Table 2 shows the bit error rate (BER) between an original watermark and an extracted watermark under different resolutions. Obviously, it can be observed that the higher the resolution is, the lower the BER is, and all BERs are sufficiently small to be negligible. Furthermore, we also observe that images with the resolutions higher than $100 \mathrm{dpi}$ seem to get comparable results.

\section{CONCLUSION}

In this study, a new digital halftone image watermarking scheme is proposed to protect "high-resolution" halftone images that are usually displayed in a computer screen or generated from printers. This is the first feature that distinguishes between our scheme and the existing methods. In 


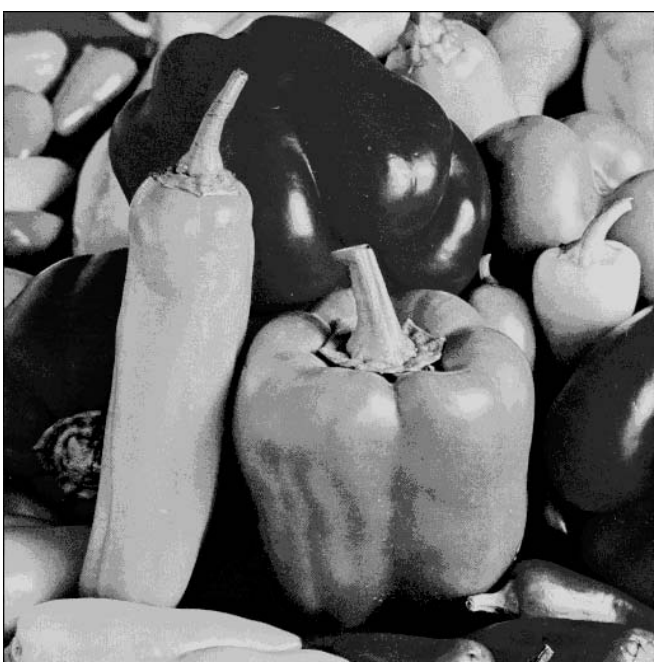

(a) Original pepper

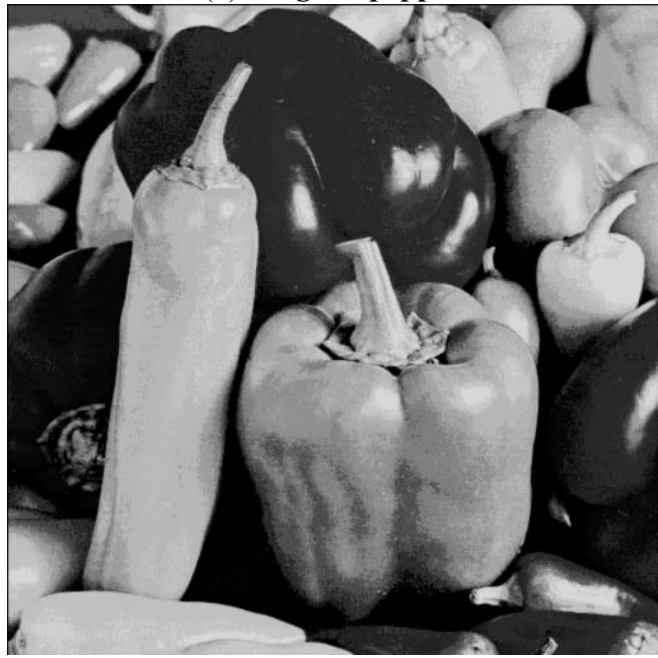

(b) Inverse halftone pepper

Fig. 4. Perceptual quality comparison between the original and the inversely high-resolution halftoned images.

addition, our scheme can be adaptive to the high-resolution digital files that will be distributed/displayed over the new device "electronic paper." On the other hand, due to the presented high-resolution halftoning process, watermarking can be directly conducted on gray-scale images by exploiting the existing robust image watermarking methods. Then, a stego halftone image is generated by means of high-resolution halftoning, and watermarks can be detected by means of high-resolution inverse halftoning. This is another feature that has not been reported in the literature. Our future work will focus on thorough evaluation of the proposed method when electronic files are attacked.
Table 2. BERs between an original watermark and an extracted watermark under different resolutions

\begin{tabular}{|c|c|c|c|}
\hline \multirow[b]{2}{*}{ Images } & \multicolumn{3}{|c|}{ Resolution } \\
\hline & 100dpi & 200dpi & 300dpi \\
\hline Splash & $1.79 e-2$ & $2.44 e-4$ & $2.44 e-4$ \\
\hline Tank & $2.01 e-2$ & 0 & 0 \\
\hline Lena & $2.59 e-2$ & 0 & 0 \\
\hline Baboon & $8.69 e-2$ & $5.90 e-3$ & $5.50 e-3$ \\
\hline Bridge & $6.24 e-2$ & $1.22 e-4$ & $1.22 e-4$ \\
\hline Clock & $3.64 e-2$ & $6.10 e-4$ & $2.44 e-4$ \\
\hline F16 & $2.87 e-2$ & 0 & 0 \\
\hline Pepper & $2.93 e-2$ & 0 & 0 \\
\hline Sailboat & $5.11 e-2$ & 0 & 0 \\
\hline Goldhill & $2.50 e-2$ & $3.66 e-4$ & $3.66 e-4$ \\
\hline
\end{tabular}

\section{REFERENCES}

[1] G. P. Crawford, "A bright new page in portable displays," IEEE Spectrum, Vol.37, pp.40-46, Oct. 2000.

[2] M. S. Fu, and O. C. Au, "Halftone image data hiding with intensity selection and connection selection," Signal Processing: Image Communication, pp.909-930, 2001.

[3] M. S. Fu, and O.C. Au, "Data hiding watermarking for halftone images," IEEE Trans. on Image Processing, Vol.11, pp.477-484, Apr. 2002.

[4] H. Z. Hel-Or, X. M. Zhang, and B. A. Wandell, "Adaptive Cluster Dot Dithering," Journal of Electronic Imaging, Vol. 8, No. 2, pp. 133-144, 1999.

[5] T. D. Kite, N. Damera-Venkata , B. L. Evans, and A. C. Bovik, "A fast, high-quality inverse halftoning algorithm for error diffused halftones," IEEE Trans. on Image Processing, Vol.9, pp. 1583-1592, Sept. 2000.

[6] D.L. Lau and G. R. Arce, Modern digital halftoning, Marcel Dekker, 2001.

[7] C. S. Lu and C. Y. Hsu, "Content-Dependent Multipurpose Watermarking Resistant Against Generalized Copy Attack," Proc. IEEE Int. Conf. on Multimedia and Expo, Taipei, Taiwan, 2004.

[8] C. S. Lu, S. W. Sun, and P. C. Chang, "Robust Mesh-based Image Watermarking with Resistance to Both Geometric Attack and WatermarkEstimation Attack," Proc. SPIE: Security, Steganography, and Watermarking of Multimedia Contents VII (EII20), 2005.

[9] M. Mese and P. P. Vaidyanathan, "Look-up table (LUT) method for inverse halftoning," IEEE Trans. on Image Processing, Vol. 10, pp. 1566-1578, Oct. 2001

[10] M. Mese and P. P. Vaidyanathan, "Tree-structured method for LUT inverse halftoning and for image halftoning," IEEE Trans. on Image Processing, Vol. 11, pp. 644-655, June 2002.

[11] S. C. Pei, and J. M. Guo, "Hybrid pixel-based data hiding and blockbased watermarking for error-diffused halftone images," IEEE Trans. on Circuits and Systems for Video Technology, Vol.13 pp.867-884, Aug. 2003.

[12] P. Sherry and A. Savakis, "Improved Techniques for Watermarking Halftone Images," Proc. IEEE Int. Conf. on Acoustics, Speech, and Signal Processing, Vol. V, pp. 1005-1008, 2004.

[13] P. W. Wong, "Inverse halftoning and kernel estimation for error diffusion," IEEE Trans. on Image Processing, Vol. 4, pp. 486-498, April 1995. 